\title{
Segregation induced by inelasticity in a vibrofluidized granular mixture
}

\author{
R. Brito, ${ }^{1,2}$ H. Enríquez, ${ }^{2}$ S. Godoy, ${ }^{1}$ and R. Soto ${ }^{1,2}$ \\ ${ }^{1}$ Departamento de Física, FCFM, Universidad de Chile, Casilla 487-3, Santiago, Chile \\ ${ }^{2}$ Departamento de Física Aplicada I and GISC, Facultad de Ciencias Físicas, Universidad Complutense, 28040 Madrid, Spain
}

(Received 14 December 2007; revised manuscript received 17 April 2008; published 3 June 2008)

\begin{abstract}
We investigate the segregation of a dense binary mixture of granular particles that only differ in their restitution coefficient. The mixture is vertically vibrated in the presence of gravity. We find a partial segregation of the species, where most dissipative particles submerge in the less dissipative ones. The segregation occurs even if one type of the particles is elastic. In order to have a complete description of the system, we study the structure of the fluid at microscopic scale (few particle diameters). The density and temperature pair distribution functions show strong enhancements with respect to the equilibrium ones at the same density. In particular, there is an increase in the probability that the more inelastic particles group together in pairs (microsegregation). Microscopically the segregation is buoyancy driven, by the appearance of a dense and cold region around the more inelastic particles.
\end{abstract}

DOI: 10.1103/PhysRevE.77.061301

\section{INTRODUCTION}

Granular matter, when externally excited, show a series of peculiar phenomena. One of them is the mixing or segregation that takes place when two or more species of different grains are put together. Depending on the control parameters and the energy injection mechanisms, grains of different size, shape, mass, or mechanical properties can mix or segregate.

Consider the particular case of a mixture of small grains and one large intruder. Here the intruder can go up [1] or down [2] — the direct and reverse Brazil nut effects, respectively, phenomenon which has been studied in many papers (see, e.g., Ref. [3] and references therein). When both species have similar sizes (but possibly different) we can select a few cases where a variety of segregation mechanisms and scenario appear [4-6]. For instance, in Ref. [7] particles of different masses, radii, and restitution coefficients are placed in a dish which is horizontally vibrated, finding complete segregation. Segregation is also found in the same geometry when the grains have different friction coefficient with the base [8]. Under horizontal swirling, radial segregation of particles of different sizes has been observed [9]. In avalanches, grains of different shape segregate in stripes [10]; in partially filled rotating drums, axial size segregation develops [11]. In two-dimensional systems under gravity, sinusoidally vibrated clustering has been observed [12]. This segregation effect can be modulated by using nonsinusoidal vibration [13].

In some of the cases mentioned above the grain species differ on the friction or restitution coefficient. However few papers have studied segregation when this is the only difference between grains. One of these cases is Ref. [14], where a mixture of spheres that only differ in friction coefficients (static, dynamic, and rolling) is horizontally vibrated. They find complete mixing - that is, no segregation-for a flat plate while segregation is only observed when the plate was slightly inclined. Therefore, these results contradict the previously mentioned ones.

In a theoretical approach, Ref. [15] constructs the hydrodynamic equations from the Boltzmann equation, finding segregation induced by inelasticity. The authors explain the phenomenon as a consequence of the temperature gradient in the system induced by inelastic collisions, and relate the con-
PACS number(s): $45.70 . \mathrm{Mg}$

centration gradient with the temperature gradient. In the same spirit, Ref. [16] studies the low density hydrodynamics of a mixture in the so-called tracer limit, i.e., where the concentration of one of the components tends to zero. Among other results, they find that the temperature ratio of both species must be a constant. This constant value was already measured by two experimental groups $[17,18]$ in two dimensions and three dimensions, respectively, and by computer simulations [19]. Generalization to high density has been done by Garzó [20].

The goal of this paper is to confirm or deny the existence of segregation induced by a inelasticity difference and characterize this phenomenon. The main tool will be molecular dynamics computer simulations of two-dimensional systems of a binary mixture kept fluidized by a vibrating base.

The structure of this paper is as follows. In Sec. II we describe the system under consideration. Section III is devoted to the macroscopic study of the system, in particular density and temperature profiles. Section IV presents a microscopic study via the pair distribution functions. Section V proposes a model that possibly explains the segregation. We conclude with Sec. VI summarizing the results of the paper.

\section{DESCRIPTION OF THE SYSTEM}

We study the effect of the difference on restitution coefficients in the segregation phenomenon, by means of molecular dynamics simulations of a bidimensional granular mixture of two types of particles, named $A$ and $B$. Grains are modeled as smooth inelastic hard disks both having the same diameter $\sigma$ and mass $m$, but differing on the normal restitution coefficient that characterizes their inelastic collisions. The restitution coefficient for $A-A$ collisions is $\alpha_{A}$, for $B-B$ collisions is $\alpha_{B}$. For the interparticle collisions $A-B$ we have taken $\alpha_{A B}=\left(\alpha_{A}+\alpha_{B}\right) / 2$. In what follows we will consider that $B$ are the most inelastic particles $\left(\alpha_{B}<\alpha_{A}\right)$.

We have taken a fixed total number of particles $N_{T}=N_{A}$ $+N_{B}$, changing the concentration of the $B$ particles. For the simulations reported in this paper, we have fixed $N_{T}=680$ disks and varied $N_{B}$ from 10 (that can be considered as a tracer limit) until 160 . The disks are placed under the action 

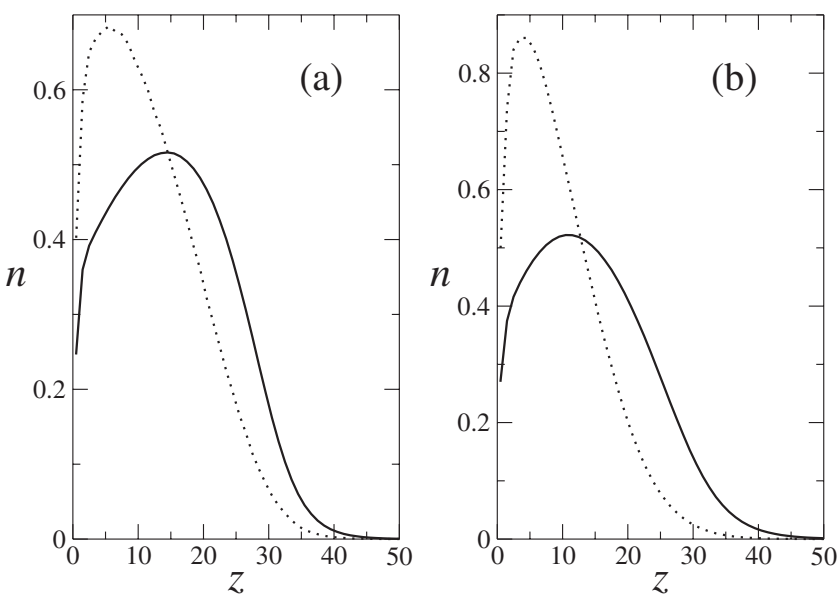

FIG. 1. Density profiles for two species, density of $A, n_{A}$ (solid line), and the rescaled density of $B, n_{B} N_{A} / N_{B}$ (dotted line). (a) Both species are inelastic with $N_{A}=670, N_{B}=10, \alpha_{A}=0.98, \alpha_{B}=0.7$. (b) $A$ is elastic while $B$ is inelastic, and $N_{A}=640, N_{B}=40$, and $\alpha_{B}=0.5$.

of a gravitational acceleration $g$ pointing downward in a rectangular box of width $L_{x}=50 \sigma$, infinite height, and with the bottom wall oscillating periodically at high frequency $\omega$ and small amplitude $A$, with a biparabolic wave form [21]. Periodic boundary conditions are used in the horizontal direction, trying to avoid the appearance of convective rolls by the influence of the walls. Under these conditions, the system reaches a stationary state with gradients in the vertical direction [22].

Units are chosen such that $\sigma=1, m=1$, and we fix the energy scale by the wall oscillation, $m(A \omega)^{2}=1$. Simulations are performed with $g=0.15$ and $A=0.01 \sigma$.

\section{MACROSCOPIC SEGREGATION}

In order to illustrate the main observed features, we report results of a simulation having a small fraction of inelastic particles $N_{B}=10$ and $\alpha_{B}=0.7$, and the rest nearly elastic: $N_{A}=670, \alpha_{A}=0.98$.

The density profiles of the two species are shown in Fig. 1(a), where we plot the number density of particles of type $A$ and $B: n_{A}(z)$ and $n_{B}(z)$. The normalization of these quantities is such that $\int_{0}^{\infty} d z \int_{0}^{L x} d x n_{A}(z)=N_{A}$ (respectively B). For plotting purposes only, $n_{B}$ is rescaled by a factor $N_{A} / N_{B}$, so in the case of no segregation both profiles would be identical. Both densities have the characteristic shape of vibrofluidized systems subject to gravity: there is an initial density increase due to the abrupt temperature drop caused by dissipation, and at higher positions, density decreases again due to gravity [22]. Density exhibits a maximum at $z \simeq 15 \sigma$ where the density $n \simeq 0.5$, so the system cannot be considered as dilute. The density profile of the more inelastic particles, $B$, is plotted as a dotted line in Fig 1(a). Its maximum is located at smaller $z$, indicating that they are closer to the bottom of the container as compared to the more elastic ones. Therefore, particles segregate although the segregation is not complete.

The temperature profiles are also highly inhomogeneous, as shown in Fig. 2(a). For both species, temperature presents
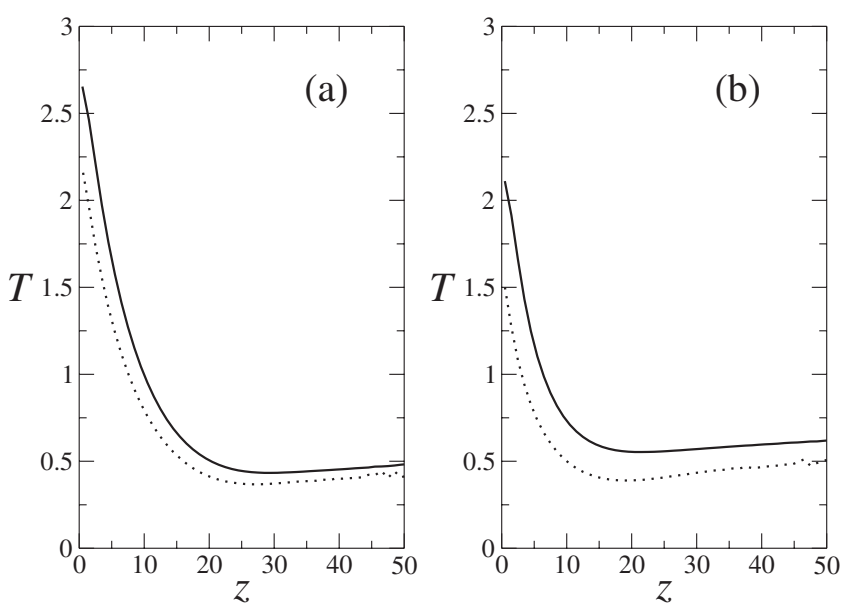

FIG. 2. Temperature profiles for two species, $T_{A}$ (solid line) and $T_{B}$ (dotted line). (a) and (b) graphics have the same parameters as in Fig. 1.

an initial abrupt drop, but later (after $z \simeq 20 \sigma$ both profiles present a linear increase with height. This phenomenon was already observed in one-component systems, and it is associated to the energy transport term, $-\mu \nabla n$, associated to density gradients, that appear in granular fluids [23]. Let us note that the maximum density does not coincide with the temperature minimum: there is a shift between these two quantities which is qualitatively described by a hydrostatic balance in the presence of gravity $[15,23]$.

The described segregation of species $A$ and $B$ is produced by their different restitution coefficients as all the other properties are the same. To study in more detail the effect of the difference of inelasticities and to understand the origin of this particular segregation, we proceed to study the limiting case in which the $A$ particles are elastic $\left(\alpha_{A}=1\right)$ and only the $B$ particles are inelastic (and consequently, collisions $A-B$ are also inelastic). In this way we also limit the parameter space, allowing to a more detailed quantitative study.

Figures 1(b) and 2(b) show the density and temperature profiles for such case, where particles of type $A$ are elastic $\left(\alpha_{A}=1\right)$ and particles $B$ are inelastic $\left(\alpha_{B}=0.5\right)$ considering $N_{B}=40$. It is observed that the main properties of the profiles are preserved, even the positive slope of $T_{A}$ despite the $A-A$ collisions being elastic. Partial segregation is again observed, where inelastic particles, $B$, sink to the bottom of the container, while elastic ones, $A$, are the majority at the upper layers of the fluid.

These results are not surprising in view of the predictions of Ref. [15], where it is argued that the segregation is produced when the particles with different restitution coefficients are immersed in a temperature gradient. The gradient is induced by the inelastic collisions, so such gradient can be created by vibrating a mixture of elastic and inelastic particles. The latter ones dissipate the energy injected by vibration creating a stationary state. The hydrodynamic description of the mixture also contains the dissipative flux $-\mu \nabla n$, and therefore it is expected that the hydrodynamic profiles of density and temperature will be equivalent to a full inelastic system.

Figure 3 shows the temperature ratios $T_{B}(z) / T_{A}(z)$ for the simulations described in Fig. 2. At low densities it was found 


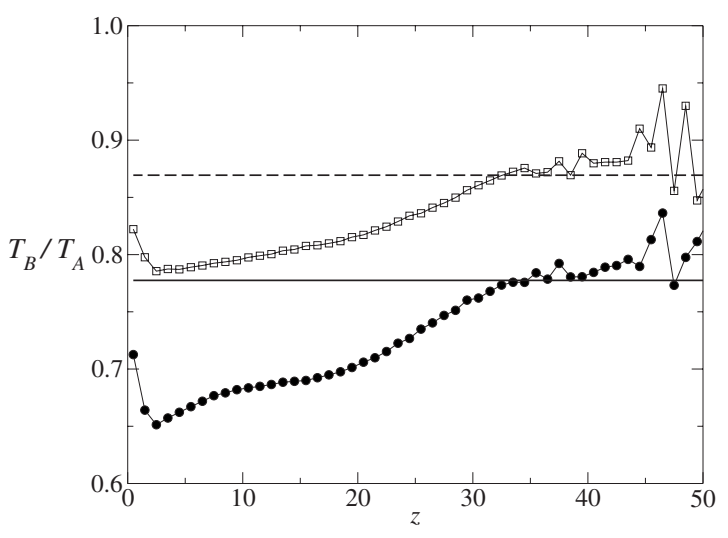

FIG. 3. Temperature ratios $T_{B}(z) / T_{A}(z)$ for the simulations described in Fig. 2. Simulation results (solid circles) and theoretical prediction in [16] (solid line) for $N_{A}=670, N_{B}=10, \alpha_{A}=0.98, \alpha_{B}$ $=0.7$. Simulation results (open squares) and theoretical prediction in [16] (dashed line) for $A$ elastic, $N_{A}=640, N_{B}=40$, and $\alpha_{B}=0.5$.

experimentally $[17,18]$ and by employing kinetic theory [16] that such temperature ratio must be constant. However, we find a nonconstant ratio in the $z$ direction that only agrees with the result of [16] at high $z$. Their prediction is valid at low densities in the tracer limit, conditions that are only reached in our case for large values of $z$. In the second case, where $A$ particles are elastic, equivalent predictions were given in $[24,25]$.

In order to quantify the segregation, a series of simulations are performed with $N_{B}$ ranging from 10 to 160 , and $\alpha_{B}$ between 0.2 and 0.9. Larger values of $N_{B}$ or smaller restitution coefficients lead to clustering as described in [26]. For each simulation we compute the segregation parameter, defined as

$$
\delta=1-\int d z n_{A}(z) n_{B}(z) / \sqrt{\int d z n_{A}^{2}(z) \int d z n_{B}^{2}(z)},
$$

where $n_{A}(z)$ and $n_{B}(z)$ are the local density, as plotted in Fig. 1 . The segregation parameter is bounded between 0 and 1 . The value $\delta=1$ corresponds to complete segregation, as $\delta$ equals 1 only if $n_{A}(z)$ and $n_{B}(z)$ do not overlap. On the contrary, $\delta=0$ means complete mixing, as this value can only be obtained if $n_{B}(z)$ is proportional to $n_{A}(z)$.

The results for $\delta$ are collected in Fig. 4 where the quantity $\delta$ is plotted versus the coefficient $\alpha_{B}$ for different values of $N_{B}$. The fact that $\delta$ is always nonvanishing confirms that the segregation exists whenever the restitution coefficients are different. Only in the case when $\alpha_{B} \rightarrow 1$ the quantity $\delta$ approaches 0 , limiting case in which there is no segregation. Note that, for each $\alpha_{B}, \delta$ increases with $N_{B}$. The results confirm that segregation is not complete as $\delta$ never becomes close to 1 . In addition, for each simulation, the center of mass of the $A$ and $B$ species are computed, $Z_{A / B}$, finding that $Z_{A}>Z_{B}$ in all cases.

It can be asked whether the observed segregation could compensate the buoyancy force experienced by lighter $B$ particles. To verify this idea, a series of simulations, keeping fixed $\alpha_{A}=1, \alpha_{B}=0.5, N_{A}=640, N_{B}=40$, and $m_{A}(A \omega)^{2}=1$, but varying $m_{B} / m_{A}$ is performed. In each simulation, the position

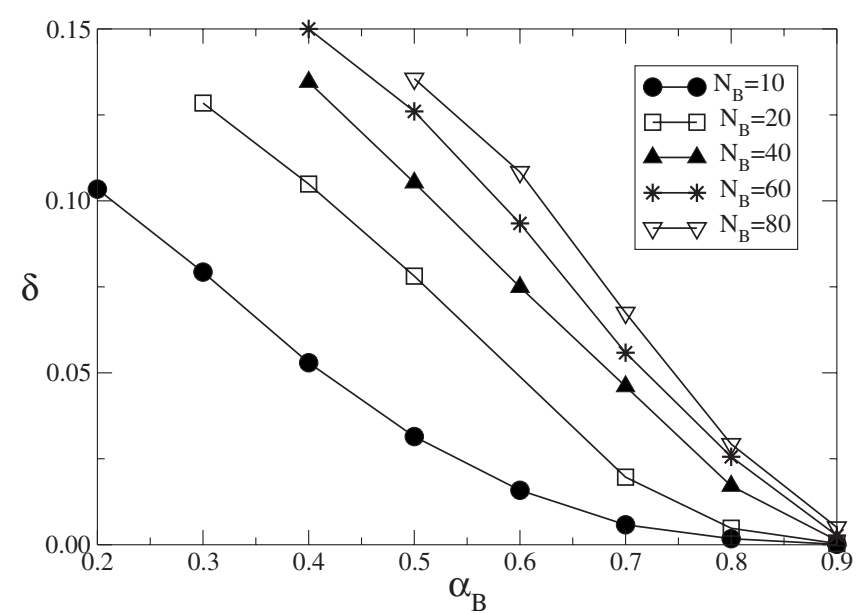

FIG. 4. Segregation parameter $\delta$ as a function of $\alpha_{B}$. Different curves correspond to various concentrations of $B$ particles. In the elastic limit $\alpha \rightarrow 1$ all curves coincide at $\delta=0$.

of the center of masses of the $A$ and $B$ species are computed, $Z_{A}, Z_{B}$. The results are presented in Fig. 5. The inelastic particles have lower center of mass if $m_{B} / m_{A}>0.37$, and therefore sinking due to dissipation wins to the buoyancy force in this range. On the contrary, buoyancy force dominates if $m_{B} / m_{A}<0.37$ and inverse segregation is obtained when $B$ particles are lighter than this threshold. The segregation parameter $\delta$ does not vanish for any value of $m_{B} / m_{A}$, indicating that there is no complete mixing even at the value of $m_{B} / m_{A}=0.37$, where both center of masses coincide. The value of $\delta$, however, is minimum at this precise mass ratio.

\section{MICROSTRUCTURE}

A snapshot of the previously studied case, $\alpha_{A}=1, \alpha_{B}$ $=0.5$, and $N_{B}=40$ is shown in Fig. 6 . The particles of type $A$ are plotted as open circles, and $B$ are black symbols. This snapshot suggest that, besides the macroscopic partial segregation characterized by $\delta$, there is also a microsegregation, where $B$ particles tend to be close to other $B$ particles, dif-

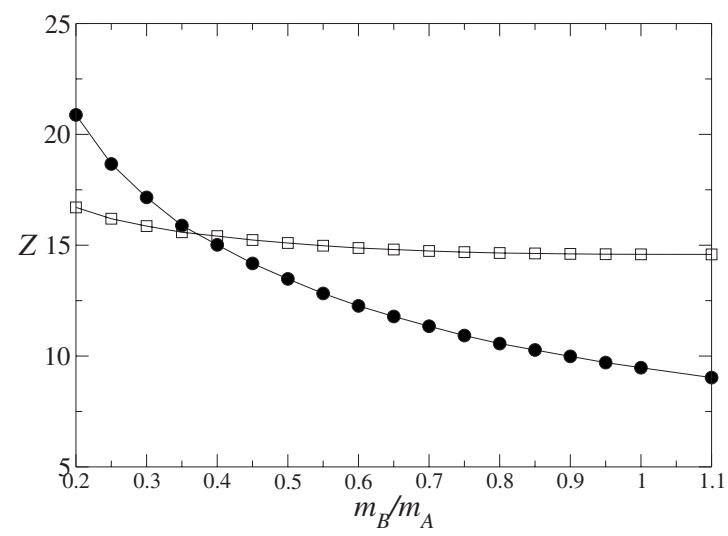

FIG. 5. Position of the center of mass of $A$ (open squares) and $B$ particles (solid circles) as a function of the relative mass of $B$ particles $m_{B} / m_{A}$. Simulation parameters are fixed to $\alpha_{A}=1, \alpha_{B}=0.5$, $N_{A}=640, N_{B}=40$, and $m_{A}(A \omega)^{2}=1$. 


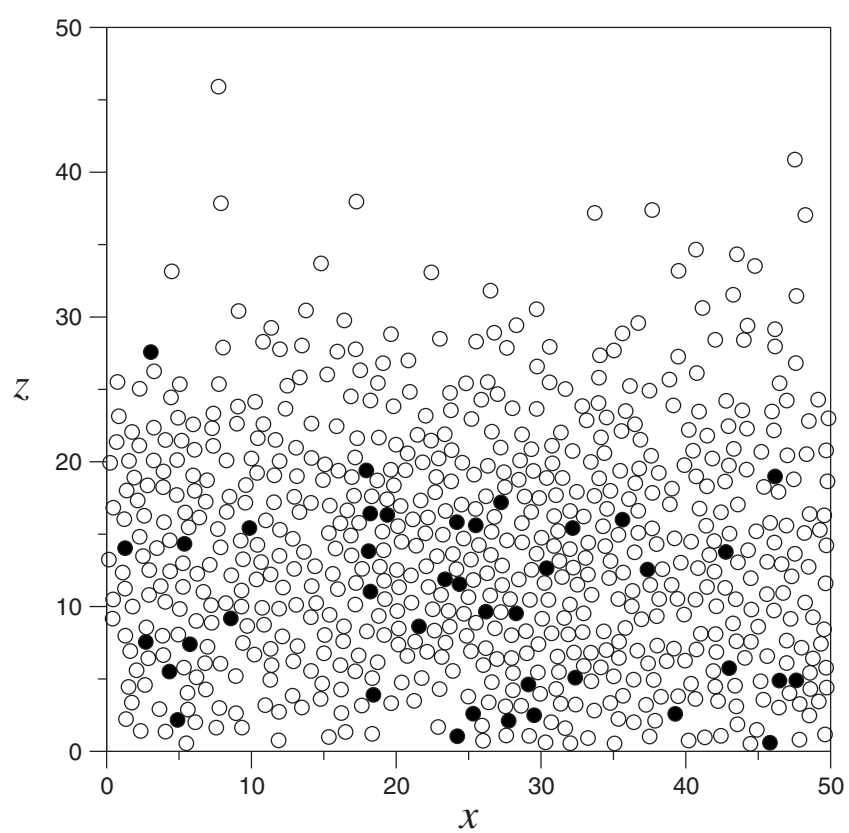

FIG. 6. Snapshot of the simulated system for $N_{B}=40, \alpha_{B}=0.5$. $A$ particles are white and $B$ particles black. The border of some particles exceed the lateral system size due to the periodic boundary conditions in this direction.

ferently as if the particles where labeled $A$ or $B$ at random. To decide if this observation is indeed true, we analyze quantitatively the system computing the pair correlation functions.

As the system is inhomogeneous in the $z$ direction, the pair correlation functions became $z$ dependent. We chose to divide the system in horizontal slabs of height $10 \sigma$. For every pair of particles, the center of mass is obtained and, according to it, their relative distance is considered for the histogram of distances associated to that particular slab. Furthermore, the pairs are classified according to the type of particles involved. Finally, the histograms are normalized as usual with the local density of each species in the slab, such that at long distances the obtained pair correlation function approaches to unity. This results in functions $g_{\mu \nu}^{s}(r)$, for the species $\mu, \nu=\{A, B\}$, and the slab $s$.

In most of the studied cases, the region of density closest to homogeneous corresponds to the second slab $(10 \sigma \leq z$ $<20 \sigma)$. In what follows we will present results only for this slab and we will suppress accordingly the superscript in the pair correlation functions. The qualitative properties for other slabs are similar to this particular slab, although the effects are reduced because at higher slabs the densities are smaller.

Figure 7 shows the density correlation function for the pairs $A A$ and $B B$ for $N_{A}=580, N_{B}=100, \alpha_{B}=0.8$, conditions in which the system develops an average density of $n$ $\simeq 0.56$. Correlation of distinct particles $A B$ have intermediate properties between $A A$ and $B B$. The main noticeable feature of these correlation functions is that the first and second peak of the $B B$ function is much larger than the $A A$ one. In other words, the large values of $g_{B B}$ at contact means that there are more $B-B$ pairs in the system that in a configuration where $A$ and $B$ particles are labeled at random. This excess number of
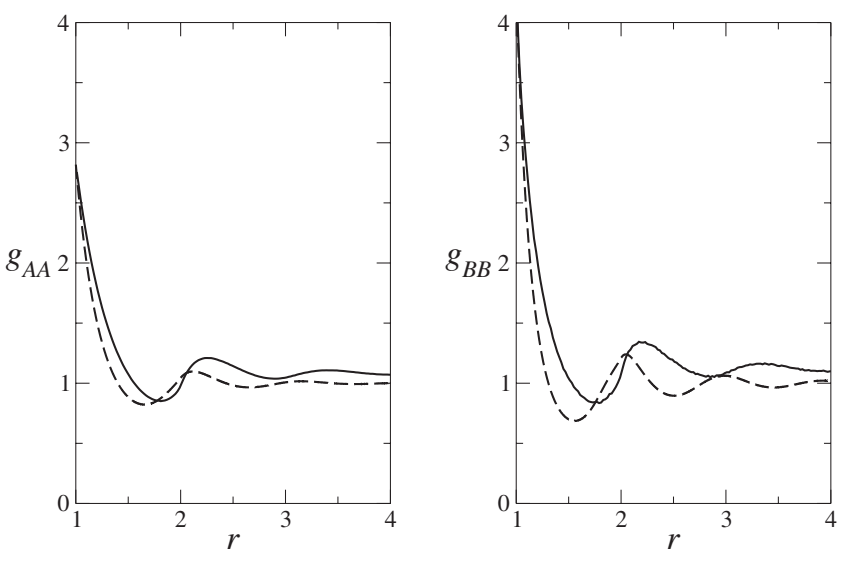

FIG. 7. Solid lines: radial distribution functions $g_{A A}(r)$ and $g_{B B}(r) . N_{B}=100, \alpha_{B}=0.8$. Dashed lines: equilibrium radial distribution function given in Ref. [28], with the density fitted to give the same value of $g_{A A}$ or $g_{B B}$ at contact (left $n_{A A}=0.636$, right $n_{B B}=0.823$ ).

$B-B$ pairs could be guessed from the Fig. 6, where one can easily locate four $B-B$ pairs.

The structure of the correlation functions in our inelastic system could resemble the radial distribution of an elastic fluid at a selected higher density. We tried to exploit this idea by comparing the granular distribution function with an elastic one by choosing an appropriate density. The way to select the density is by adjusting the value of the pair distribution function at contact. We take this criterion inspired by the kinetic theory of dense fluids, where the pair distribution function at contact is used to improve the Boltzmann equation including certain correlations. With such procedure we find different fitting densities for $A A$ and $B B$ pairs, being their values: $n_{B B}=0.823$ and $n_{A A}=0.636$. Surprisingly, none of them (not even the elastic) agree with the average density in the system, $n \simeq 0.56$. This means that the dissipative particles, type $B$, are able to modify the structural properties of the $A$ particles, which we have chosen to be elastic. The fitted elastic pair correlation functions are plotted as dashed lines in Fig. 7. Despite the fitting procedure, large discrepancies are observed between our correlation functions and the elastic ones. Other fitting procedures could have been selected, but none of them produce an agreement of the correlation functions in all its range.

The inelastic correlation function shows a strong enhancement of the first peak, followed by a second, and at most a third peak, reflecting a short-range structure of few particle diameters. However, the location of the peaks differs from the elastic correlation function, indicating changes in the microstructure. The fast decay of the correlation function in inelastic systems has been observed in homogeneously heated inelastic gases as well [27]. This analysis confirms the existence of a microsegregation of particles of type $B$.

In addition, the pair correlation function at contact $\chi$ $=g\left(\sigma^{+}\right)$is computed for the pairs $A A, A B$, and $B B$. The values of $\chi$ grow when increasing $N_{B}$ and decreasing $\alpha_{B}$, obeying that $\chi_{B B}>\chi_{A B}>\chi_{A A}$. Finally, let us remark that $\chi_{B B}$ can reach very high values. For instance, at $N_{B}=160$ and $\alpha_{B}$ $=0.5, \chi_{B} \simeq 60$, despite that the average total density is only $n \simeq 0.5$. 


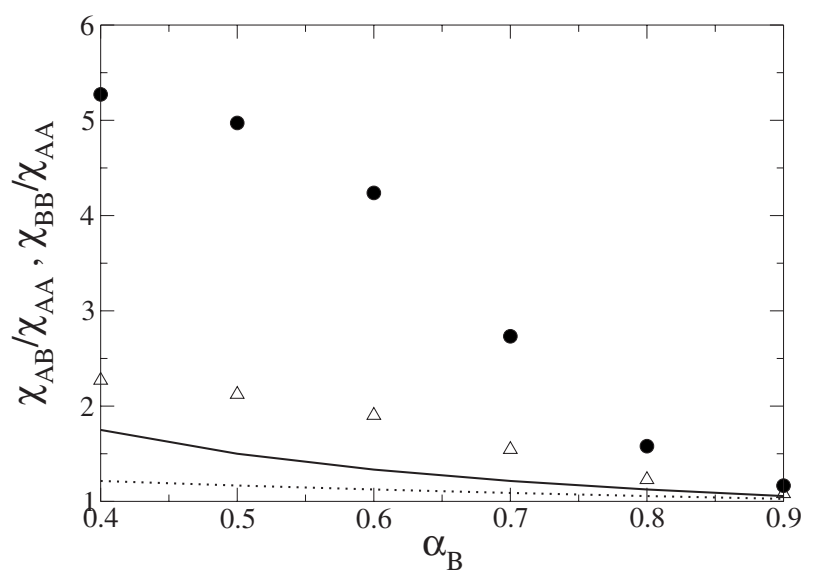

FIG. 8. Contact pair correlation function quotients as a function of the restitution coefficient. $\chi_{B B} / \chi_{A A}$ results from simulations (filled circles) and the Enskog theoretical value (solid line); and $\chi_{A B} / \chi_{A A}$ results from simulations (open triangles) and the Enskog theoretical value (dotted line). Here $N_{B}=120$.

It has been proposed [29], based on an Enskog-like kinetic model that, due to the smaller outgoing velocity after a collision, particles stay longer in the close vicinity, when the restitution coefficient is less than one. These arguments lead to an enhancement of the pair correlation function in terms of the restitution coefficient $\alpha$, as

$$
\chi(\alpha)=\frac{1+\alpha}{2 \alpha} \chi_{0} .
$$

Here $\chi_{0}$ is the pair correlation function of elastic particles at the same density that are given by the Verlet-Levesque [30] and Carnahan-Starling [31] factors for two and three dimensions.

To test if this prediction is valid we have plotted in Fig. 8 the ratios $\chi_{B B} / \chi_{A A}$ against $\alpha_{B}$ and $\chi_{A B} / \chi_{A A}$ against $\alpha_{A B}$ and compared them with the factors $\left(1+\alpha_{B}\right) /\left(2 \alpha_{B}\right)$ and $(1$ $\left.+\alpha_{A B}\right) /\left(2 \alpha_{A B}\right)$, respectively. Dividing by $\chi_{A A}$ we eliminate the $\chi_{0}$ factor in Eq. (2). The figure indicates that the Enskog model is not enough to describe the high values of the pair correlation when one inelastic particle is present, as was already quoted by its author [29]. A possible origin of this effect are recollisions, that are not taken into account in Enskog's theory, a phenomenon that is pronounced in granular systems. Inelastic particles, after a collision, separate at a slower speed, increasing the possibility of having a collision with a third particle, approaching it again the original pair. In some way, inelasticity increases the so-called cage effect in liquids.

Besides density correlations induced by $B$ particles, they also create a local decrease of temperature because their collisions are inelastic. We have computed the radial temperature of particles of type $\mu$ located at a distance $r$ of a $\nu$ particle: $T_{\mu \nu}(r)$. Figure 9 shows these radial temperature functions. Their asymptotic values are the average temperatures of $A$ and $B$ particles in the slab $10 \sigma<r<20 \sigma$. Besides the fact that $B$ particles are colder than $A$ particles, they produce a local decrease of temperature both for $A$ and $B$ particles. This effect is more pronounced when increasing the

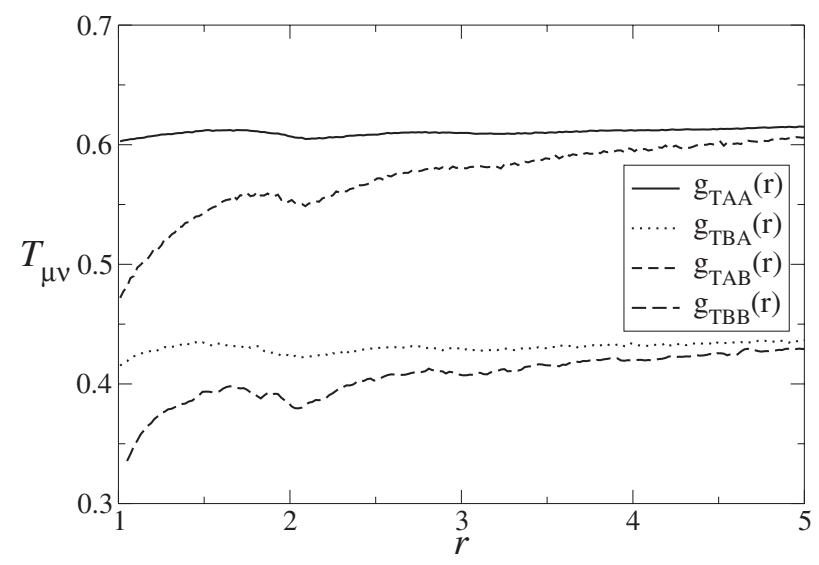

FIG. 9. Radial temperatures $T_{\mu \nu}$ of a particle of type $\mu$ around a particle of type $\nu . N_{B}=40, \alpha_{B}=0.5$.

inelasticity but almost independent of the concentration of $B$ particles, whose main effect is to reduce globally the temperature. As before this is a local effect that extends for about $2 \sigma$.

\section{ORIGIN OF THE GLOBAL SEGREGATION}

So far we have argued that $B$ particles locally modify the structure of $B$ particles (inelastic) and also of $A$ particles (elastic). For instance, Fig. 7 shows that the density around $B$ particles is higher than around $A$ particles. The excess mass around $B$ particles with respect to $A$ particles is defined as

$$
\delta m=\int_{\sigma}^{\infty} d r 2 \pi r\left[\rho_{B}(r)-\rho_{A}(r)\right],
$$

where $\rho_{\mu}(r)$ is the total density around a particle of type $\mu$. The excess mass is positive for all values of $N_{A}, N_{B}$, and restitution coefficient $\alpha_{B}$. Similarly, the local temperature around a $B$ particle is lower than the local temperature around an $A$ particle (see Fig. 9).

These two results imply that around a $B$ particle is developed a dense and cold region, that we call a cold droplet. The characteristic size of the droplet is microscopic; in agreement with the plots of Sec. IV, must be about 2-3 $\sigma$. The development of a cold droplet around each $B$ particle increases their effective mass and also, due to the lower temperature, decreases the pressure. This mechanism could be similar (at the local level) to the clustering instability described in [32]. In our case the unstable process does not continue as the energy injection at the vibrating wall breaks the droplets. These droplets must be seen as dynamical objects, that form and evanesce continuously. Once the cold droplet is formed around $B$ the buoyancy force is weaker than its effective weight and therefore there is a net force that tends to sink the cluster and consequently the $B$ particle.

This continuous sink of the $B$ particles is responsible for the macroscopic segregation described in Sec. III.

\section{SUMMARY AND CONCLUSIONS}

The main conclusion is that different restitution coefficients alone create segregation in a binary mixture vertically 
vibrated. The restitution coefficients must be considered, in addition to the usual material properties (mass ratio and diameter ratio) in order to describe accurately the segregation. The effect of the inelasticity is such that the most inelastic particles sink to the bottom of the container while the less inelastic ones rise to the top. Segregation is not complete, however, but only partial. The density profiles of each species shows a maximum which is located in a different position depending on the inelasticities. Concerning the temperatures, most dissipative particles have a lower temperature than most elastic ones, both having the characteristic shape of vibrofluidized system (fast cooling away from the moving boundary followed by a heating that grows linearly with the distance).

The segregation effects presented here also appear by vibrating a mixture of elastic and inelastic particles. Again inelastic particles migrate to the bottom of the container and elastic one prefer the upper part. The temperature distribution also looks similar to the full inelastic mixture.

Besides the macroscopic segregation there is also segregation at the microscopic scale. With the word microscopic we refer to properties at distances of few particles diameters. In our case we find a notorious increase of the probability of finding two inelastic particles together as compared with the less inelastic or elastic ones. This enhancement cannot be described by only considering kinematic properties. Our guess is that dynamic correlations are required to properly describe these correlations.
Finally, we propose a mesoscopic explanation to the segregation: the most inelastic particles induce locally a region of high density and low temperature, resembling a cold droplet that falls in a gravitational field. The droplet is created by the dissipation in a way that resembles the clustering instability of the granular gases.

The effect of the different restitution coefficients may act in an opposite direction as the usual Brazil nut effect. For instance, consider a vibrated granular fluid and insert a large (or light) intruder than tends to move upward. If the intruder is very dissipative, it will move downwards, as we have described in this paper. Which is the final position? Which force does finally win? A partial answer is presented in Fig. 5, where we show the competition between the buoyancy force and the sinking effect due to dissipation. Moreover, could two large an inelastic intruders come together by the effect of inelasticity alone, as shown in Sec. IV of the present paper? Further research is needed in order to answer these questions.

\section{ACKNOWLEDGMENTS}

We want to thank J. M. R. Parrondo for very useful comments. R.B. was supported by the Spanish Projects MOSAICO, Contract Nos. FIS2004-271 and UCM/PR34/0715859. The research was supported by Fondecyt Grant Nos. 1061112, 1070958, and 7070301 and Fondap Grant No. 11980002 .
[1] A. Rosato, K. J. Strandburg, F. Prinz, and R. H. Swendsen, Phys. Rev. Lett. 58, 1038 (1987).

[2] A. P. J. Breu, H.-M. Ensner, C. A. Kruelle, and I. Rehberg, Phys. Rev. Lett. 90, 014302 (2003).

[3] A. Kudrolli, Rep. Prog. Phys. 67, 209 (2004).

[4] D. A. Huerta and J. C. Ruiz-Suárez, Phys. Rev. Lett. 92, 114301 (2004); 93, 069901(E) (2004).

[5] M. Schröter, S. Ulrich, J. Kreft, J. B. Swift, and H. L. Swinney, Phys. Rev. E 74, 011307 (2006).

[6] D. V. Kharkar, J. J. McCarthy, and J. M. Ottino, Chaos 9, 594 (1999).

[7] P. M. Reis and T. Mullin, Phys. Rev. Lett. 89, 244301 (2002).

[8] M. P. Ciamarra, A. Coniglio, and M. Nicodemi, Phys. Rev. Lett. 94, 188001 (2005).

[9] T. Schnautz, R. Brito, C. A. Kruelle, and I. Rehberg, Phys. Rev. Lett. 95, 028001 (2005).

[10] H. A. Makse, S. Havlin, P. R. King, and H. E. Stanley, Nature (London) 386, 379 (1997).

[11] K. M. Hill and J. Kakalios, Phys. Rev. E 49, R3610 (1994).

[12] D. A. Sanders, M. R. Swift, R. M. Bowley, and P. J. King, Phys. Rev. Lett. 93, 208002 (2004).

[13] L. T. Lui, Michael R. Swift, R. M. Bowley, and P. J. King, Phys. Rev. E 75, 051303 (2007).

[14] L. Kondic, R. R. Hartley, S. G. K. Tennakoon, B. Painter, and R. P. Behringer, Europhys. Lett. 61, 742 (2003).

[15] D. Serero, I. Goldhirsch, S. H. Noskowick, and M.-L. Tan, J. Fluid Mech. 554, 237 (2006), the case of different restitution coefficients is treated in Sec. 5.1.
[16] J. J. Brey, M. J. Ruiz-Montero, and F. Moreno, Phys. Rev. E 73, 031301 (2006).

[17] R. D. Wildman and D. J. Parker, Phys. Rev. Lett. 88, 064301 (2002).

[18] K. Feitosa and N. Menon, Phys. Rev. Lett. 88, 198301 (2002).

[19] D. Paolotti, C. Cattuto, U. Marini Bettolo Marconi, and A. Puglisi, Granular Matter 5, 75 (2003).

[20] V. Garzó, Europhys. Lett. 75, 521 (2006); e-print arXiv:0803.2588.

[21] R. Soto, Phys. Rev. E 69, 061305 (2004).

[22] E. L. Grossman, T. Zhou, and E. Ben-Naim, Phys. Rev. E 55, 4200 (1997).

[23] R. Ramírez and R. Soto, Physica A 322, 73 (2003).

[24] Ph. A. Martin and J. Piasecki, Europhys. Lett. 46, 613 (1999).

[25] A. Barrat, E. Trizac, Granular Matter 4, 57 (2002).

[26] B. Meerson, T. Pöschel, and Y. Bromberg, Phys. Rev. Lett. 91, 024301 (2003).

[27] I. Pagonabarraga, E. Trizac, T. P. C. van Noije, and M. H. Ernst, Phys. Rev. E 65, 011303 (2001).

[28] J. Kolafa, S. Labík, and A. Malijevský, Phys. Chem. Chem. Phys. 6, 2335 (2004); see also http://www.vscht.cz/fcs/ software/hsmd/ for molecular dynamics results of $g(r)$.

[29] J. F. Lutsko, Phys. Rev. E 63, 061211 (2001).

[30] L. Verlet and D. Levesque, Mol. Phys. 46, 969 (1982).

[31] N. F. Carnahan and K. E. Starling, J. Chem. Phys. 51, 635 (1969).

[32] I. Goldhirsch and G. Zanetti, Phys. Rev. Lett. 70, 1619 (1993). 